\title{
Tissue Doppler Imaging: An Overview
}

\author{
Mohamed Nashnoush ${ }^{1, *}$, Muneeza Sheikh ${ }^{2}$ and Chopra Chirag ${ }^{3}$ \\ 2 University of Toronto; c.chopra@mail.utoronto.ca \\ 3 Dalhousie University; mh537521@dal.ca \\ * Correspondence: mnashnoush@dal.ca; Tel.: +19022666010
}

\begin{abstract}
Tissue Doppler Imaging (TDI) is a non-invasive, echocardiographic imaging technique that measures myocardial motion velocity throughout the cardiac cycle using Doppler principles. While conventional techniques assess blood flow velocity by sensing high-frequency, lowamplitude signals from small, fast-moving blood cells, TDI uses the same Doppler principles to measure high-amplitude, lower-velocity signals of myocardial tissue. A literature review was conducted to survey and review studies investigating limitations, strengths, physical principles, novel methods, applications in diseased states, and prognostic capabilities of TDI. Articles were further screened for inclusion, and those deemed ineligible or irrelevant to the scope of the review were discarded. In total, 19 studies were included in the qualitative synthesis. TDI is shown to be an effective method for detailed quantification of cardiac function. It provides an early means of diagnosing cardiac dysfunction and is a valid prognostic indicator for various forms of heart disease. TDI's versatility and precision allows clinicians to predict the clinical course of disease, leading to early intervention and the selection of targeted care management plans for many cardiac pathologies. Despite imaging limitations like angle dependence and incapacity for passive and active motion differentiation, further investigation continues to reveal novel TDI methodologies that advance the scope of this imaging technique.
\end{abstract}

Keywords: TDI, Doppler, Echocardiography

\section{Introduction}

TDI is a supplemental echocardiographic tool that is principally used for cardiovascular diagnostic imaging [1]. Derived from conventional echocardiographic techniques and rooted in Doppler principles, TDI provides quantitative measures of myocardial function by capturing systolic and diastolic myocardial motion [2]. TDI indicates the rate of movement of a specific point of myocardial tissue by measuring the point's movement relative to the transducer [3]. Over time, this measurement can yield displacement and absolute distance moved by the point being imaged. These quantities can be utilized as clinical indices of various functional and anatomical abnormalities including heart failure, acute myocardial infarction, and hypertension [1,2,4]. Due to TDI's quantitative objectivity and heightened sensitivity, the technique is now considered a benchmark for assessing subclinical myocardial dysfunctions expediting a diagnosis in the case of an imminent myocardial infarction $[1,2]$.

TDI differs from traditional Doppler techniques by assessing high-amplitude, lowvelocity signals compared to the high-frequency, low amplitude signals native to traditional techniques [5]. TDI-derived prognostic markers include annular systolic ( $\left.\mathrm{S}^{\prime}\right)$, early diastolic $\left(E^{\prime}\right)$, and atrial diastolic contraction $\left(A^{\prime}\right)$ velocities, constituting the three waveforms which compose the cardiac cycle [4].

The growing efficacy of TDI's analytical advancements and diagnostic capabilities in the past two decades renders it a powerful prognosticator and a reliable tool to measure the efficacy of potential clinical interventions [1]. The clinically significant utility and versatility of TDI is embodied in the various modes available - including color mode, 
pulse-wave, and 3-D modes - allowing for multiple imaging specifications and insights [4]. Thus, TDI proves to be a promising clinical imaging feature with prognostic value and diagnostic potential [5].

\section{Materials and Methods}

Peer-reviewed studies published in the past eight years utilizing a contemporary form of TDI were considered in this study. All papers reviewed were documented in English, and studies discussing multiple novel TDI applications were specifically noted. Diversity in the specialization and implementation was considered in addition to considering a diverse target population of specific TDI applications.

The databases used in this review include PubMed, Cochrane Library, MEDLINE, Google Scholar, Canadian Medical Association Journal, Canadian Journal of Medical Sonography, and Novanet. Additional records were identified through other sources, which were filtered for duplicates. In the PubMed database, MeSh terms such as "Elasticity Imaging Techniques," "Ultrasonography," "Physics," and "Patient Care Management" were utilized to refine the search. To increase the relevance of search results, Boolean operators, such as "AND" and "OR" were utilized. Furthermore, a population inclusion filter was applied to ensure that the populations studied were composed of adults at a minimum age of 19. An adult age group filter was applied because of the lack of substantiated and rigorous manuscripts investigating pediatric and neonatal TDI echocardiographic applications. The review also employed the Cochrane handbook for systematic reviews of interventions to assess randomized controlled trials (RCTs), cohort studies, longitudinal studies, and meta-analyses [6].

The title and abstract of all studies were first evaluated for relevance and applicability by applying the exclusion criteria of data duplication, recency, publication status, and insufficient rigour. Full-text articles were assessed and excluded based on insufficient controlled diagnostic criteria, outdated TDI technique and a lack of relevance to echocardiographic application. 


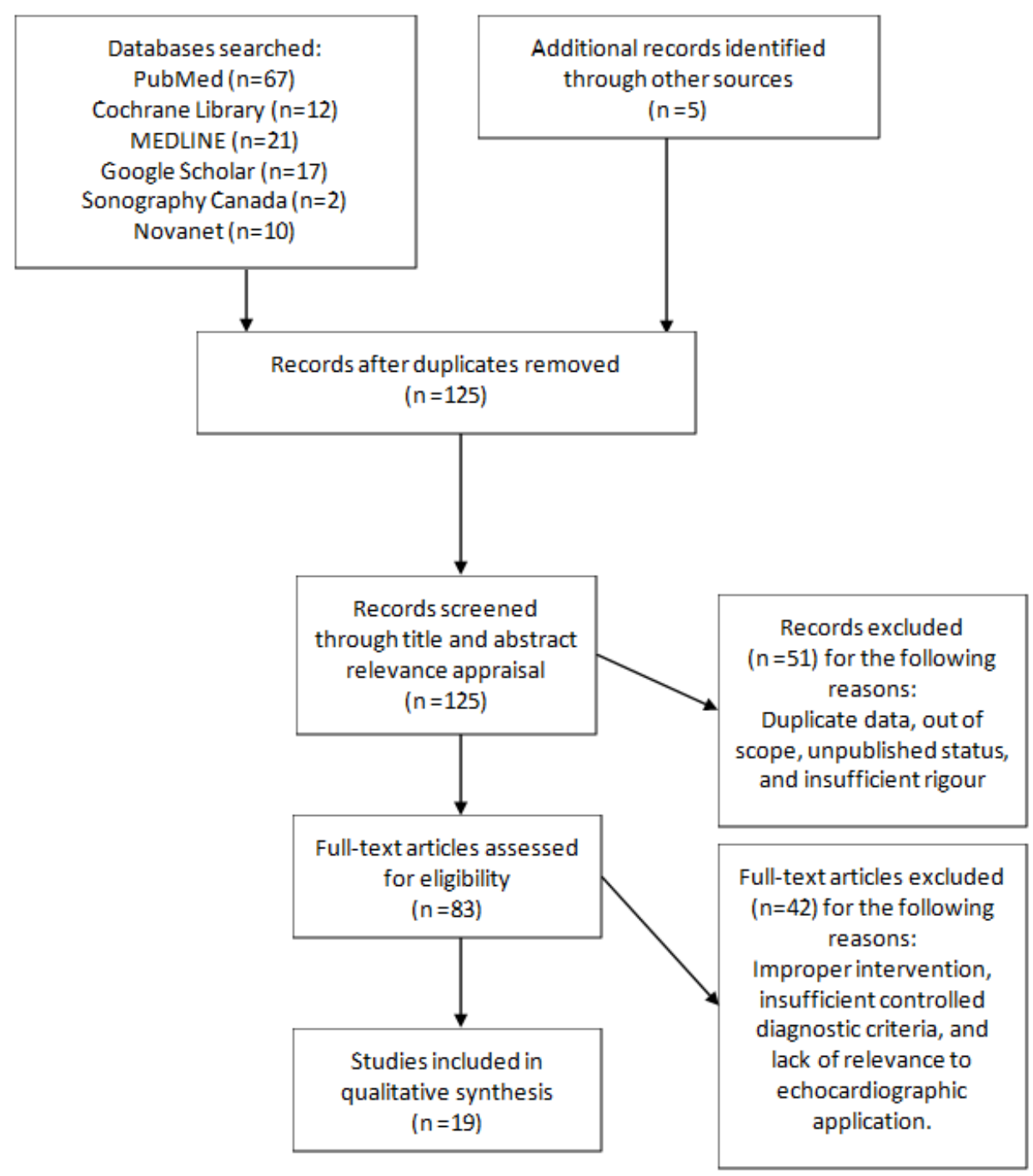

Figure 1. Flow diagram.

\section{Discussion}

TDI is primarily used in echocardiography as a prognosticator of myocardial dysfunction and as a supplementary diagnostic tool to evaluate systolic and diastolic function [7]. TDI applies fundamental Doppler principles to capture low frequency, high amplitude signals of myocardial wall motion $[7,8]$. Unlike conventional Doppler imaging, TDI removes the high-pass filter to accentuate low myocardial velocities and can be applied in three primary ways: color mode, pulse wave, and 3-D mode [8].

\subsection{Color Mode TDI}

With color TDI, a color-coded representation of myocardial velocities is superimposed on 2D grayscale to reveal the direction and velocity of myocardial motion $[8,9]$. Some advantages of Color TDI include the elevated detail resolution and the capacity to evaluate multiple structures in a single view [7].

Color TDI signal processing represents mean velocities, whereas pulse-wave TDI reports the higher peak velocities from the selected sample volume [7]. An aliasing-free velocity scale that is sensitive to both lower velocity recordings and higher myocardial velocities should be selected to optimize the image quality $[7,8,9,10]$.

In a study with 61 subjects, color TDI proved to appraise regional left ventricular function objectively [11]. The normal and abnormal segmental endocardial velocity responses to dobutamine stress were evaluated with color TDI and compared to routine 
visual inspection of segmental function with two-dimensional grayscale images [8,9]. Segmental peak endocardial velocities measured by TDI increased significantly in all segments in the control group, while endocardial velocities were significantly lower at peak stress in those who developed infarcted segments [9]. These values were equivalent to those obtained with the wall motion score index, demonstrating that color TDI can allow for the detection of subclinical myocardial dysfunctions $[8,9]$.

\subsection{Pulse-Wave TDI}

Pulse-wave TDI (PW TDI) is available in most contemporary ultrasound systems and is utilized to measure peak myocardial velocities [7]. The general set-up of PW TDI is virtually identical to a typical pulse-wave, where the gate is opened to $2-5 \mathrm{~mm}$ and directed on the mitral annulus at the lateral and medial sites from the apical four-chamber view $[8,10]$. The apical four-chamber window is the optimal view in capturing long-axis ventricular motion due to the longitudinally oriented endocardial fibers providing a zero Doppler angle of incidence $[8,9,10]$. PW TDI has the advantage of a superior temporal resolution, but lacks the capacity to image multiple segments in a single view [9].

Several indices are acquired from PW TDI that have both clinical and prognostic implications [10]. These indices include:

Sa (S'): systolic myocardial velocity seen above the baseline [7].

Ea (E'): early diastolic myocardial relaxation displayed below the baseline [7].

Aa (A'): atrial contraction myocardial velocity seen below the baseline [7].

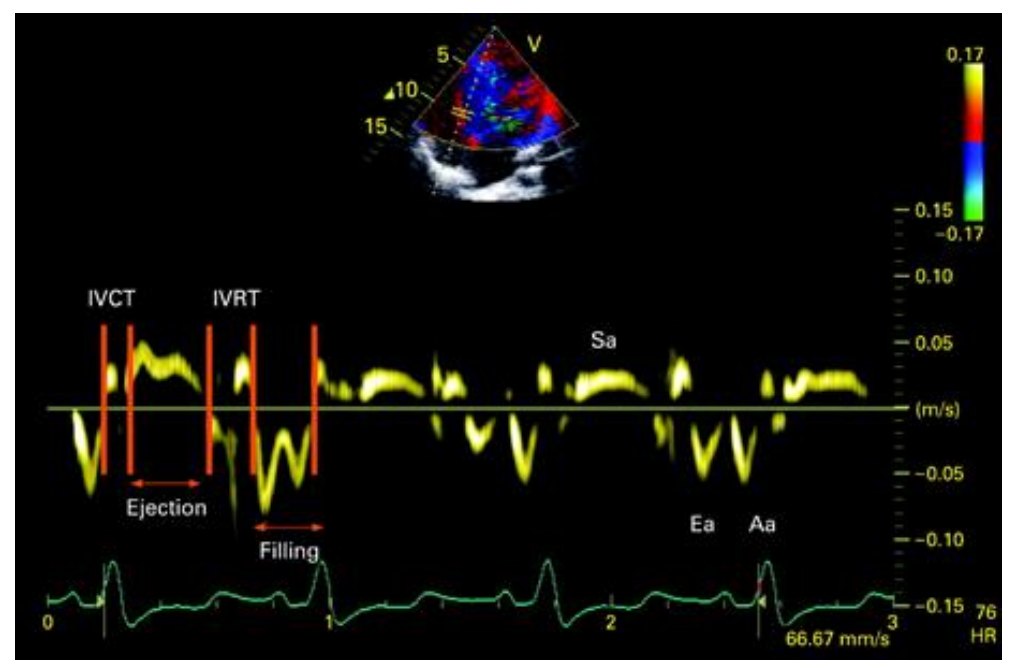

Figure 2. A normal PW TDI waveform recorded at the septal mitral valve annulus. The stages of the myocardial cycle encompass isovolumic contraction time (IVCT), ejection, isovolumic relaxation time (IVRT) and filling [7].

The sample volume placement and size prove to be critical parameters in attaining accurate Doppler tissue tracings $[7,8]$. Subtle changes in the location of the sample volume outside the annulus could result in erroneous recordings [9]. According to a recent retrospective study, manually adjusting the sample volume size such that it becomes proportional to the annular motion and degree of excursion increases the accuracy of recorded velocities and waveforms [7]. This would be accomplished by using an approximate $3.5 \mathrm{~mm}$ sample volume to account for less excursion in the septal annulus and utilizing an approximate $5.0 \mathrm{~mm}$ sample volume to capture greater motion in the lateral annulus $[7,10]$.

There are two major limitations of PW TDI, which include the angle dependence and the failure to discriminate between active and passive motion $[6,10]$. The zero-degree angle assumption may prove problematic in patients with a body habitus that limits appropriate transducer alignment, or when intervening pleural pathologies limit visualization requiring the use of an alternative window [9]. Translation and tethering of 
myocardial tissue may occasionally be interpreted as true contractility by the ultrasound system, limiting the reliability of the recorded indices [10].

\subsection{3-D TDI}

Recently, a three-dimensional (3-D) TDI imaging modality - triplane TDI - has become available [11]. It is based on real-time volumetric imaging that allows the acquisition of pyramidal datasets from one cardiac cycle and projects the image as an online real-time display $[10,11,12]$. Its ability to visualize three-dimensional cardiac structures and dynamic motion images from any spatial point of view provides an overarching appraisal of cardiac chamber volumes and their functions [11,12]. The advantage 3-D TDI provides in visualizing cardiac anatomy in transthoracic examinations grants detailed analysis of the mitral valve anatomy in patients with mitral regurgitation (MR) $[13,14]$. However, it lacks the ability to present high image quality and resolve between two closely timed events $[15,16]$. The time required for data acquisition and analysis is significant, often resulting in temporal and spatial misregistration $[17,18]$.

Authors should discuss the results and how they can be interpreted from the perspective of previous studies and of the working hypotheses. The findings and their implications should be discussed in the broadest context possible. Future research directions may also be highlighted.

Table 1. Evaluation of risk of bias in articles relevant to discussion.

\begin{tabular}{|c|c|c|c|c|c|c|}
\hline Reference & Title & Author/year & $\begin{array}{c}\text { Study } \\
\text { Design }\end{array}$ & $\begin{array}{l}\text { Sample } \\
\text { Size (n) }\end{array}$ & Findings & $\begin{array}{c}\text { Risk of } \\
\text { Bias }\end{array}$ \\
\hline
\end{tabular}

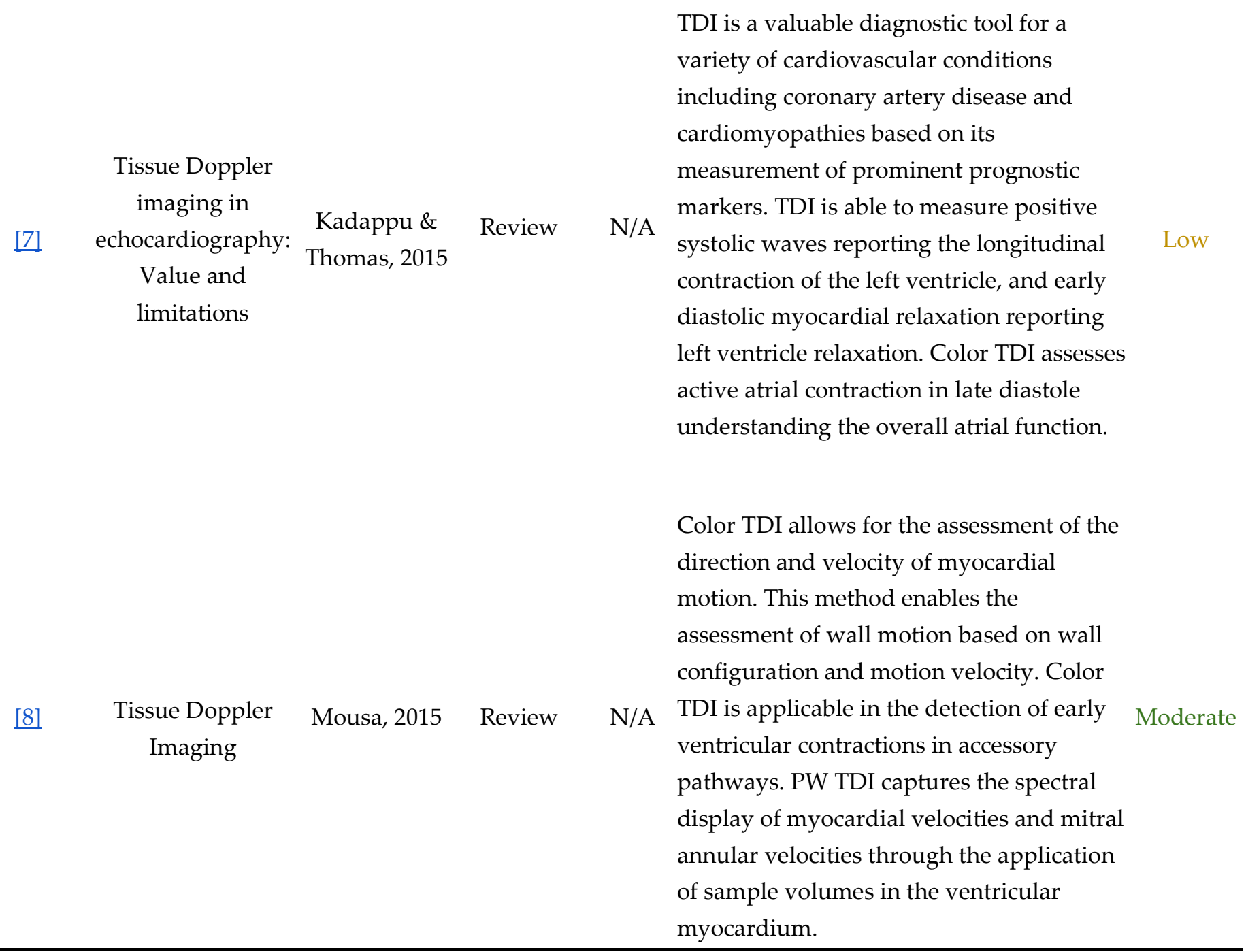




$\begin{array}{ccc}\begin{array}{c}\text { Assessment of } \\ \text { myocardial function }\end{array} & \\ \text { in neonates using } & \text { Negrine et al., } & \begin{array}{c}\text { Primary } \\ \text { Research }\end{array} \\ \text { tissue Doppler } & 2012 & \text { Article } \\ \text { imaging } & & \end{array}$

Reference values for

[10] and tissue Doppler imaging in pediatric Da 2015 Article echocardiography pulse wave Doppler

The prognostic value of left ventricular systolic function measured by tissue Doppler imaging in septic shock

\section{Real-time 3D} echocardiography and tissue Doppler echocardiography in

\section{Primary \\ Weng et al., Research 2012 \\ Article}

61

They found that the use of TDI for measuring left ventricular dysfunction, which has also served as an independent predictor of septic shock mortality amongst patients.
Recent technological

$\begin{array}{ccc}\text { advancements in } & \text { Dave et al., } & \text { Review } \\ \text { cardiac ultrasound } & 2018 \\ \text { imaging } & \end{array}$

TDI successfully provided quantifiable velocity measures of myocardial function of both right and left ventricles during cardiac assessments amongst preterm

43 neonates. Authors report the velocities were easier to assess amongst preterm infants 30-36 weeks compared to those very preterm (i.e., $<30$ weeks). One waveform provided both systolic and diastolic function information of newborn myocardial function.

They were successful in providing more standardized $\mathrm{Z}$ scores for pulse wave consideration body size.
They used three-dimensional echocardiography and TDI to evaluate the right ventricle function while also assessing the right ventricle ejection fraction with a diagnosis of right ventricle infarction.
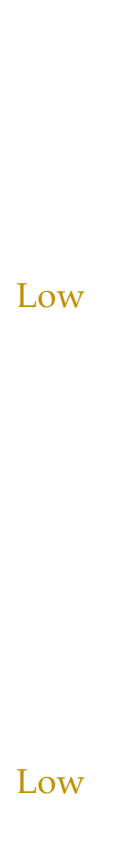

Low<smiles>CCC</smiles>

Low

TDI utilizes low-pass filters instead of the high pass filters of conventional doppler imaging to assess low frequency, high

N/A amplitude signals characteristic of Moderate myocardial contraction. Modes include color or pulsed waves, which differ based on sampling volume capacities and 
limitations for velocity measurements.

Pulsed wave-TDI can measure

instantaneous velocity but only one

sample volume can be assessed at a time,

whereas color-TDI is faster due to

simultaneous volumetric assessments but

is more prone to angular errors.

Low: Low risk of bias

Moderate: Sound for a nonrandomized study but not comparable with a rigorous randomized trial

\section{Conclusions}

The prognostic potential of TDI as a quantitative marker for global and regional myocardial function is promising. Unlike conventional ultrasound techniques, TDI is unhindered by tissue interfaces between the transducer and site of imaging $[7,10]$. In conjunction with conventional echocardiography, its ability to analyze and assess systolic and diastolic functions is useful in evaluating various cardiovascular diseases [11]. TDI measures the characteristic high-amplitude, low-velocity signals of myocardial tissue motion and can be adjusted to various modes capable of imagining radial and longitudinal motions but with varying limitations $[7,18,19]$. The growing efficacy of this precise, non-invasive, versatile, and reproducible imaging modality allows clinicians to predict the clinical course of disease, leading to early intervention and individualized patient care for many cardiac pathologies [19]. Despite the robustness of TDI, emerging clinical applications face imaging limitations, including the necessity of an insonation angle that is parallel or within 15 degrees of the area of interest and the inability of TDI to differentiate passive motion from active motion $[7,17]$. Areas of further investigation can include novel clinical applications of TDI, such as assessments of diaphragmatic muscle motion imaging in critically-ill patients [1].

Author Contributions: Conceptualization, M.N. and M.S.; methodology, M.N.; formal analysis, M.N.; investigation, M.N.; resources, M.N.; data curation, M.N. and M.S.; writing - original draft preparation, M.N.; writing-review and editing, M.N., M.S. and C.C. All authors have read and agreed to the published version of the manuscript.

Funding: This research received no external funding.

Institutional Review Board Statement: Not applicable.

Informed Consent Statement: Not applicable.

Acknowledgments: The authors acknowledge the assistance/support of Dr. Erin Lushman for providing critical feedback and suggestions in the development of the manuscript.

Conflicts of Interest: The authors declare no conflict of interest.

\section{References}

1. Méndez-Abad P, Zafra-Rodríguez P, Lubián-López S, Benavente-Fernández I. Myocardial Function Maturation in Very-LowBirth-Weight Infants and Development of Bronchopulmonary Dysplasia. Front Pediatr. 2020 Jan 17;7:556.

2. Rajesh GN, Raju D, Nandan D, Haridasan V, Vinayakumar D, Muneer K, Sajeev CG, Babu K, Krishnan MN. Echocardiographic assessment of right ventricular function in inferior wall myocardial infarction and angiographic correlation to proximal right coronary artery stenosis. Indian Heart Journal. 2013 Sep 1;65(5): 522-8.

3. Nowicki A, Dobruch-Sobczak K. Introduction to ultrasound elastography. J Ultrason. 2016 Jun;16(65):113-24.

4. Rist HJ, Mauch M. Quantified TDI elastography of the patellar tendon in athletes. Sportverletzung Sportschaden: Organ der Gesellschaft fur Orthopadisch-Traumatologische Sportmedizin. 2012 Mar;(26)1:27-32. 
5. Brekke B, Nilsen LC, Lund J, Torp H, Bjastad T, Amundsen BH, Stoylen A, Aase SA. Ultra-high frame rate tissue Doppler imaging. Ultrasound in medicine \& biology. 2014 Jan 1;40(1):222-31.

6. Moher D, Shamseer L, Clarke M, Ghersi D, Liberati A, Petticrew M, Shekelle P, Stewart LA. Preferred reporting items for systematic review and meta-analysis protocols (PRISMA-P) 2015 statement. Systematic reviews. 2015 Dec 1;4(1):1.

7. Kadappu KK, Thomas L. Tissue Doppler imaging in echocardiography: Value and limitations. Heart, Lung and Circulation. 2015 Mar 1;24(3):224-33.

8. Mousa HK. Tissue Doppler Imaging. J Cardiol Curr Res. 2015, 3(4):00113.

9. Negrine RJ, Chikermane A, Wright JG, Ewer AK. Assessment of myocardial function in neonates using tissue Doppler imaging. Archives of Disease in Childhood-Fetal and Neonatal Edition. 2012 Jul 1;97(4):F304-6.

10. Dallaire F, Slorach C, Hui W, Sarkola T, Friedberg MK, Bradley TJ, Jaeggi E, Dragulescu A, Har RL, Cherney DZ, Mertens L. Reference values for pulse wave Doppler and tissue Doppler imaging in pediatric echocardiography. Circulation: Cardiovascular Imaging. 2015 Feb;8(2):e002167.

11. Weng L, Liu YT, Du B, Zhou JF, Guo XX, Peng JM, Hu XY, Zhang SY, Fang Q, Zhu WL. The prognostic value of left ventricular systolic function measured by tissue Doppler imaging in septic shock. Critical Care. 2012 Jun 1;16(3):R71.

12. Sert A, Aypar E, Pirgon O, Yilmaz H, Odabas D, Tolu I. Left ventricular function by echocardiography, tissue Doppler imaging, and carotid intima-media thickness in obese adolescents with nonalcoholic fatty liver disease. The American journal of cardiology. 2013 Aug 1;112(3):436-43.

13. Mahjoub Y, Benoit-Fallet H, Airapetian N, Lorne E, Levrard M, Seydi AA, Amennouche N, Slama M, Dupont H. Improvement of left ventricular relaxation as assessed by tissue Doppler imaging in fluid-responsive critically ill septic patients. Intensive care medicine. 2012 Sep 1;38(9):1461-70.

14. Wu V, Chyou JY, Chung S, Bhagavatula S, Axel L. Evaluation of diastolic function by three-dimensional volume tracking of the mitral annulus with cardiovascular magnetic resonance: comparison with tissue Doppler imaging. Journal of Cardiovascular Magnetic Resonance. 2014 Dec 1;16(1):71.

15. Correale M, Totaro A, Ieva R, Ferraretti A, Musaico F, Di Biase M. Tissue Doppler imaging in coronary artery diseases and heart failure. Current cardiology reviews. 2012 Feb 1;8(1):43-53.

16. Vitarelli A, Mangieri E, Terzano C, Gaudio C, Salsano F, Rosato E, Capotosto L, D'Orazio S, Azzano A, Truscelli G, Cocco N. Three-dimensional echocardiography and 2D-3D speckle-tracking imaging in chronic pulmonary hypertension: Diagnostic accuracy in detecting hemodynamic signs of right ventricular (RV) failure. Journal of the American Heart Association. 2015 Mar 19;4(3):e001584.

17. Kidawa M, Chiżyński K, Zielińska M, Kasprzak JD, Krzeminska-Pakula M. Real-time 3D echocardiography and tissue Doppler echocardiography in the assessment of right ventricle systolic function in patients with right ventricular myocardial infarction. European Heart Journal-Cardiovascular Imaging. 2013 Oct 1;14(10):1002-9.

18. Dave JK, Mc Donald ME, Mehrotra P, Kohut AR, Eisenbrey JR, Forsberg F. Recent technological advancements in cardiac ultrasound imaging. Ultrasonics. 2018 Mar 1;84:329-40.

19. Soilemezi E, Savvidou S, Sotiriou P, Smyrniotis D, Tsagourias M, Matamis D. Tissue Doppler imaging of the diaphragm in healthy subjects and critically ill patients. American Journal of Respiratory and Critical Care Medicine. 2020 Oct 1;202(7):1005-12. 